\title{
Efficient performance analysis of ATM buffer systems by using the spectral analysis of rate matrices
}

\author{
Kristiaan Wuyts* and René K. Boe** \\ University of Ghent \\ SYSTeMS, Technologiepark-Zwijnaarde 9, B-9052 Ghent, Belgium, \\ tel: +32 92645655 and +32 92645658 , \\ fax: +3292645840 , \\ email: Kristiaan.Wuyts@rug.ac.be and Rene.Boel@rug.ac.be
}

\begin{abstract}
The goal of this paper is an algorithm to find the CLR and cell delay characteristics for realistic ATM buffer systems with a large number $N$ of reasonably complicated Markov modulated Bernoulli sources with $M$ states per source. Matrix geometric methods have already reduced the complexity of finding these QoS measures by using rate matrices. However the dimensions of the rate matrices still grow exponentially with $N$. In ATM the number of sources at the input of these buffer systems is often so large that straightforward application of matrix geometric methods is impossible, since a set of $(N-1) M^{2 N}$ nonlinear equations would have to be solved to obtain the rate matrices. This paper presents a technique, based on the spectral analysis of the rate matrix, which makes computing the CLR for large $N$ feasible. Using the Kronecker product structure of the blocks of the transition matrix, the problem of finding the eigenvalues of the rate matrix can be reduced to solving a set of $N+1$ non-linear scalar equations in the eigenvalue $s$ of the rate matrix and in $N$ dummy variables. Only 1 equation contains all variables; the other $N$ equations contain 1 dummy variable and $s$. Most of the eigenvalues can be found by using repeated substitution in the set of equations, but for some unstable roots we need a combination of repeated substitution and Powell's Direction Set Method minimizing a squared error function. The eigenvectors of the rate matrix are obtained by solving as many sets of $M$ linear scalar equations

\footnotetext{
* The results presented in this paper have been obtained partially within the framework of the Belgian Program on Interuniversity Attraction Poles, initiated by the Belgian State, Prime Minister's Office, Science Policy Programming, and partially within the framework of the ITA-MULTIMEDIA project of the IWT (Flemish Institute for Science and Technology). The scientific responsibility rests with its authors. R. K. Boel is supported by the Fund for Scientific Research - Flanders (F.W.O.) as Senior Research Associate. K. Wuyts was partly supported by a grant from the B.O.F. - University of Ghent.
}

The original version of this chapter was revised: The copyright line was incorrect. This has been corrected. The Erratum to this chapter is available at DOI: 10.1007/978-0-387-35353-1_28 
as there are different eigenvalues of the rate matrix. It is possible to further reduce the complexity of the calculations by only considering eigenvectors corresponding to the most significant eigenvalues.

\section{INTRODUCTION}

Any ATM-network contains several buffer systems (such as statistical multiplexers, rate adapters, switching elements, ...) where congestion can occur. If some sources put more cells in a buffer than the server can take out, and if this situation holds for a fairly long time, the buffer can become full and newly arriving cells are lost. Also the delay in a filled buffer is usually a substantial amount of the total delay cells experience when they travel through the network. Therefore anyone who is interested in the performance of an ATM-network certainly wants to look at the Cell Loss Ratio or the cell delay characteristics (CTD, CDV , ...) in 1 buffer for a given arrival traffic. Which performance measure has to be computed depends on the traffic type. For real-time video (such as for video-conferencing) good delay characteristics are more valuable than a small CLR, whereas for the transmission of data (e.g. via $\mathrm{ABR}$ ) it is more important to receive the information correctly and without losses.

The performance criteria described above are stationary measures, which have the advantage that they can be computed from the equilibrium distribution of the buffer occupancy. This is the probability that, starting from any initial condition at $t=0$, the buffer will contain $n$ cells after an infinite long time. Also non-stationary measures can be used to describe the performance of a network (such as the correlation between cell losses during a burst of arriving cells), but these fall outside the scope of this paper.

In this paper we are mostly interested in the effects on the buffer occupancy of VBR traffic (video and data) which can be modelled by Markov Modulated processes (such as MMBP). These processes have also the advantage that they can approximate long range dependence over several orders of magnitude of the time scale, e.g. (Robert and Le Boudec "Can..." 1995) or (Robert and Le Boudec "Stoch..." 1995). The results in this paper can be used for the dimensioning of buffers, the study of resource allocation, construction of flow control schemes, etc. Flow control will be the topic of a future paper: the effects of flow control will be considered there by introducing one or more thresholds in the buffer. As soon as the buffer occupancy exceeds a threshold, the sources (usually buffer systems upwards of the arrival traffic stream) are requested to lower the rates at which they send cells to the buffer.

Several methods for the computation of the equilibrium distribution of the buffer occupancy have already been developed as in (Blondia and Casals 1992), (Ye and Li 1994), (Xiong and Bruneel 1996) and (Naoumov et al. 1996). The Matrix Geometric Method (Neuts 1981) is widely used because of its numerical stability and its computational efficiency. In (Neuts 1981) it is 
developed for Quasi-Birth-and-Death processes and infinite buffers, and in (Latouche and Ramaswami 1993) the authors propose an algorithm which reduces the complexity of calculating the rate matrix in a logarithmic way. In a modified form this matrix geometric method can be applied for finite buffers (Hajek 1982). In (Wuyts and Boel 1996) the authors propose an extension of the matrix geometric method for a finite buffer with several arrival streams, where the QBD-structure is no longer valid.

Unfortunately, even in the infinite buffer case the method of (Wuyts and Boel 1996) still involves the computation of $N-1$ rate matrices of dimension $M^{N} \times M^{N}$ yielding a set of $(N-1) M^{2 N}$ non-linear equations. In an ATM-network $N$ can be very large (e.g. $N=20$ ) such that the computation of the rate matrices becomes impossible. The main contribution of this paper is to present a technique, based on the spectral analysis of a rate matrix, which makes computing the CLR for large $N$ possible, i.e. for realistic ATM arrival streams. The advantage of the spectral analysis of this matrix is that the matrix itself does not have to be computed and the method makes use of the inner Kronecker product structure of matrices $\mathbf{A}_{\mathbf{i}}$ (corresponding to the arrival of $i$ cells in 1 slot) in order to compute the $(N-1) M^{N}$ eigenvalues as efficiently as possible. Finding the eigenvalues of the rate matrix can then be reduced to solving a set of $N+1$ nonlinear scalar equations in the eigenvalue $s$ of the rate matrix and $N$ dummy variables. The computation complexity can be further reduced by computing only dominating eigenvalues and their corresponding eigenvectors. Finding heuristic rules for deciding which eigenvalues are dominant (e.g. in the computation of the CLR) is a topic of current research. The method above can be compared with (Mitrani and Chakka 1995), where an analogous spectral method is described for a continuous time infinite buffer problem. This paper can therefore be seen as an extension to the discrete time finite and infinite buffer case with several independent Markovian arrival streams, implying a Kronecker product structure.

In section 2 we describe a model for the VBR arrival traffic at an ATM buffer. In section 3 we introduce an algorithm for the spectral decomposition of the rate matrices and in section 4 the eigenvalues and corresponding left eigenvectors are used to compute the equilibrium equation. The algorithm is used to find some numerical results for a B-ISDN example in section 5 and section 6 shows briefly how to extend the algorithm to the finite buffer case.

\section{MODEL}

Consider one buffer in an ATM buffer system (such as a statistical multiplexer, a switching element, ...) with $N$ independent Markov modulated Bernoulli sources at the input and 1 server at the output. The server removes one cell per time slot from the buffer if it is not empty. The transition matrix of the modulating Markov process for source $i$ is given by an $M \times M$-dimensional matrix $\mathbf{Q}_{\mathbf{i}} ; \mathbf{P}_{\mathbf{i}}$ is an $M \times M$-dimensional diagonal matrix with diagonal ele- 
ments equal to the probability that source $i$ will send a cell during a slot in which its state is $j$.

Since we want to study the behaviour of the buffer occupancy, we need the Markov process $Y_{k}=\left(B_{k}, S_{k}(1), \ldots, S_{k}(N)\right) \in\{0, \ldots, B\} \times\{1, \ldots, M\} \times \ldots \times$ $\{1, \ldots, M\} ; B_{k}$ denotes the number of cells in the buffer during slot $k$ and $S_{k}(i)$ represents the state of the modulating Markov process of source $i$ during slot $k$. The collection of all states $Y_{k}$ with $B_{k}=b$ cells in the buffer is called the "level" $b$.

Let us consider for now only the infinite buffer case (later we show briefly how the technique must be modified for the finite buffer case). The transition matrix $\mathbf{P}$ of $Y_{k}$ is:

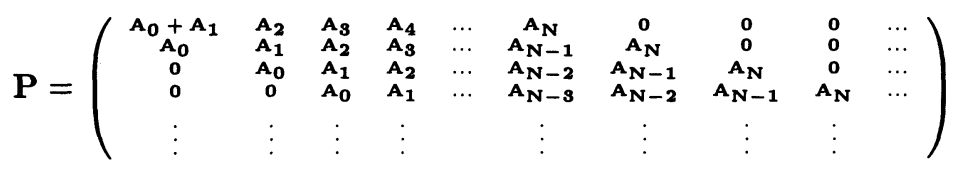

where each $\mathbf{A}_{\mathbf{n}}$ is an $\left(M^{N} \times M^{N}\right)$-dimensional matrix, constructed as follows:

$$
\left\{\begin{aligned}
\mathbf{A}_{\mathbf{N}}= & \mathbf{P}_{\mathbf{1}} \mathbf{Q}_{\mathbf{1}} \otimes \mathbf{P}_{\mathbf{2}} \mathbf{Q}_{\mathbf{2}} \otimes \ldots \otimes \mathbf{P}_{\mathbf{N}} \mathbf{Q}_{\mathbf{N}} \\
\mathbf{A}_{\mathbf{N}-1}= & \left(\mathbf{I}-\mathbf{P}_{\mathbf{1}}\right) \mathbf{Q}_{\mathbf{1}} \otimes \mathbf{P}_{\mathbf{2}} \mathbf{Q}_{\mathbf{2}} \otimes \ldots \otimes \mathbf{P}_{\mathbf{N}} \mathbf{Q}_{\mathbf{N}} \\
& +\mathbf{P}_{\mathbf{1}} \mathbf{Q}_{\mathbf{1}} \otimes\left(\mathbf{I}-\mathbf{P}_{\mathbf{2}}\right) \mathbf{Q}_{\mathbf{2}} \otimes \mathbf{P}_{\mathbf{3}} \mathbf{Q}_{\mathbf{3}} \otimes \ldots \otimes \mathbf{P}_{\mathbf{N}} \mathbf{Q}_{\mathbf{N}} \\
& +\ldots \\
& +\mathbf{P}_{\mathbf{1}} \mathbf{Q}_{\mathbf{1}} \otimes \mathbf{P}_{\mathbf{2}} \mathbf{Q}_{\mathbf{2}} \otimes \ldots \otimes \mathbf{P}_{\mathbf{N}-\mathbf{1}} \mathbf{Q}_{\mathbf{N}-\mathbf{1}} \otimes\left(\mathbf{I}-\mathbf{P}_{\mathbf{N}}\right) \mathbf{Q}_{\mathbf{N}} \\
\vdots & \\
\mathbf{A}_{\mathbf{0}}= & \left(\mathbf{I}-\mathbf{P}_{\mathbf{1}}\right) \mathbf{Q}_{\mathbf{1}} \otimes\left(\mathbf{I}-\mathbf{P}_{\mathbf{2}}\right) \mathbf{Q}_{\mathbf{2}} \otimes \ldots \otimes\left(\mathbf{I}-\mathbf{P}_{\mathbf{N}}\right) \mathbf{Q}_{\mathbf{N}}
\end{aligned}\right.
$$

and $\otimes$ denotes the Kronecker product.

The structure in (2) consists of sums of Kronecker products because the arrival stream consists of independent Markov modulated Bernoulli processes. This can be easily seen if one considers a simple example, e.g. $N=2$ sources with $M=2$ states per source:

$\mathbf{P}_{\mathbf{i}}=\left(\begin{array}{cc}p_{1}(i) & 0 \\ 0 & p_{2}(i)\end{array}\right), \quad \mathbf{Q}_{\mathbf{i}}=\left(\begin{array}{cc}q_{1,1}(i) & q_{1,2}(i) \\ q_{2,1}(i) & q_{2,2}(i)\end{array}\right)$

The transition matrix $\mathbf{P}$ now consists of 3 diagonals, containing the matrices $\mathbf{A}_{\mathbf{0}}, \mathbf{A}_{\mathbf{1}}$ and $\mathbf{A}_{\mathbf{2}}$. $\left(\mathbf{A}_{0}\right)_{1,1}$ is the probability that no cells arrive at the buffer during slot $k$, source 1 will be in state 1 during slot $k+1$ and source 2 will be in state 1 during slot $k+1$ under the condition that source 1 is in state 1 during slot $k$ and that source 2 is in state 1 during slot $k$. This probability equals $\left(1-p_{1}(1)\right) q_{1, \mathbf{1}}(1)\left(1-p_{1}(2)\right) q_{1,1}(2)=\left[\left(\mathbf{I}-\mathbf{P}_{\mathbf{1}}\right) \mathbf{Q}_{\mathbf{1}}\right]_{1,1} \cdot\left[\left(\mathbf{I}-\mathbf{P}_{\mathbf{2}}\right) \mathbf{Q}_{\mathbf{2}}\right]_{1,1}$. 
In the same way we prove that:

$$
\begin{aligned}
\left(\mathbf{A}_{\mathbf{0}}\right)_{1,2} & =\left(1-p_{1}(1)\right) q_{1, \mathbf{1}}(1)\left(\mathbf{1}-p_{\mathbf{1}}(2)\right) q_{1,2}(2) \\
& =\left[\left(\mathbf{I}-\mathbf{P}_{\mathbf{1}}\right) \mathbf{Q}_{\mathbf{1}}\right]_{1,1} \cdot\left[\left(\mathbf{I}-\mathbf{P}_{\mathbf{2}}\right) \mathbf{Q}_{\mathbf{2}}\right]_{1,2} \\
\left(\mathbf{A}_{\mathbf{0}}\right)_{1, \mathbf{3}} & =\left(1-p_{1}(1)\right) q_{1,2}(1)\left(1-p_{\mathbf{1}}(2)\right) q_{1,1}(2) \\
& =\left[\left(\mathbf{I}-\mathbf{P}_{\mathbf{1}}\right) \mathbf{Q}_{\mathbf{1}}\right]_{1,2} \cdot\left[\left(\mathbf{I}-\mathbf{P}_{\mathbf{2}}\right) \mathbf{Q}_{\mathbf{2}}\right]_{1,1} \\
\left(\mathbf{A}_{\mathbf{0}}\right)_{1,4} & =\left(\mathbf{1}-p_{1}(1)\right) q_{1,2}(1)\left(1-p_{1}(2)\right) q_{1,2}(2) \\
& =\left[\left(\mathbf{I}-\mathbf{P}_{\mathbf{1}}\right) \mathbf{Q}_{\mathbf{1}}\right]_{1,2} \cdot\left[\left(\mathbf{I}-\mathbf{P}_{\mathbf{2}}\right) \mathbf{Q}_{\mathbf{2}}\right]_{1,2} \\
\left(\mathbf{A}_{\mathbf{0}}\right)_{2, \mathbf{1}} & =\left(1-p_{1}(1)\right) q_{1,1}(1)\left(1-p_{\mathbf{2}}(2)\right) q_{2, \mathbf{1}}(2) \\
& =\left[\left(\mathbf{I}-\mathbf{P}_{\mathbf{1}}\right) \mathbf{Q}_{\mathbf{1}}\right]_{1,1} \cdot\left[\left(\mathbf{I}-\mathbf{P}_{\mathbf{2}}\right) \mathbf{Q}_{\mathbf{2}}\right]_{2,1} \\
\left(\mathbf{A}_{\mathbf{0}}\right)_{2, \mathbf{1}} & =\left(\mathbf{1}-p_{1}(\mathbf{1})\right) q_{1,1}(1)\left(1-p_{\mathbf{2}}(2)\right) q_{2,2}(2) \\
& =\left[\left(\mathbf{I}-\mathbf{P}_{\mathbf{1}}\right) \mathbf{Q}_{\mathbf{1}}\right]_{1,1} \cdot\left[\left(\mathbf{I}-\mathbf{P}_{\mathbf{2}}\right) \mathbf{Q}_{\mathbf{2}}\right]_{2,2} \\
\left(\mathbf{A}_{\mathbf{0}}\right)_{2, \mathbf{1}} & =\left(1-p_{\mathbf{1}}(1)\right) q_{1,2}(1)\left(1-p_{\mathbf{2}}(2)\right) q_{2, \mathbf{1}}(2) \\
& =\left[\left(\mathbf{I}-\mathbf{P}_{\mathbf{1}}\right) \mathbf{Q}_{\mathbf{1}}\right]_{1,2} \cdot\left[\left(\mathbf{I}-\mathbf{P}_{\mathbf{2}}\right) \mathbf{Q}_{\mathbf{2}}\right]_{2, \mathbf{1}} \\
\left(\mathbf{A}_{\mathbf{0}}\right)_{2, \mathbf{1}} & =\left(1-p_{1}(1)\right) q_{1,2}(1)\left(1-p_{\mathbf{2}}(2)\right) q_{2,2}(2) \\
& =\left[\left(\mathbf{I}-\mathbf{P}_{\mathbf{1}}\right) \mathbf{Q}_{\mathbf{1}}\right]_{1,2} \cdot\left[\left(\mathbf{I}-\mathbf{P}_{\mathbf{2}}\right) \mathbf{Q}_{\mathbf{2}}\right]_{2, \mathbf{2}}
\end{aligned}
$$

This can be repeated for the third and fourth row of $\mathbf{A}_{\mathbf{0}}$ and for $\mathbf{A}_{\mathbf{1}}$ and $\mathbf{A}_{\mathbf{2}}$. This proves that in Kronecker product notation $\mathbf{A}_{\mathbf{i}}$ can be written as:

$$
\left\{\begin{array}{l}
\mathbf{A}_{2}=\mathbf{P}_{\mathbf{1}} \mathbf{Q}_{\mathbf{1}} \otimes \mathbf{P}_{\mathbf{2}} \mathbf{Q}_{\mathbf{2}} \\
\mathbf{A}_{\mathbf{1}}=\left(\mathbf{I}-\mathbf{P}_{\mathbf{1}}\right) \mathbf{Q}_{\mathbf{1}} \otimes \mathbf{P}_{\mathbf{2}} \mathbf{Q}_{\mathbf{2}}+\mathbf{P}_{\mathbf{1}} \mathbf{Q}_{\mathbf{1}} \otimes\left(\mathbf{I}-\mathbf{P}_{\mathbf{2}}\right) \mathbf{Q}_{\mathbf{2}} \\
\mathbf{A}_{\mathbf{0}}=\left(\mathbf{I}-\mathbf{P}_{\mathbf{1}}\right) \mathbf{Q}_{\mathbf{1}} \otimes\left(\mathbf{I}-\mathbf{P}_{\mathbf{2}}\right) \mathbf{Q}_{\mathbf{2}}
\end{array}\right.
$$

Thus the Kronecker product expresses that the Markov process remembers the state of every individual source during a slot; this requires 4 states for the case of two 2-state sources.

\section{SPECTRAL DECOMPOSITION OF THE RATE MATRICES}

The stationary probability distribution of $\mathbf{P}$ is the infinite row vector $\underline{\pi}=$ $\left[\underline{\pi}_{0} \underline{\pi}_{1} \ldots\right]$ where every $\underline{\pi}_{b}$ is a row vector with dimension $M^{N}$ (corresponding to level $b$ ); assuming the average arrival rate $\sum r_{i}<1, \underline{\pi}$ is the unique solution of

$$
\left\{\begin{array}{l}
\underline{\pi} \cdot \mathbf{P}=\underline{\pi} \\
\sum_{i=0}^{\infty} \underline{\pi}_{i} \cdot \underline{e}=1
\end{array}\right.
$$


In (Wuyts and Boel 1996) it is shown that $\underline{\pi}$ has the following generalized matrix geometric structure:

$$
\underline{\pi}_{n}=\underline{\pi}_{n-1} \boldsymbol{R}_{1}+\underline{\pi}_{n-2} \boldsymbol{R}_{2}+\ldots+\underline{\pi}_{n-N+1} \boldsymbol{R}_{N-1} \quad n=N-1, N, \ldots
$$

where the rate matrices $\boldsymbol{R}_{1}, \boldsymbol{R}_{2}, \ldots, \boldsymbol{R}_{N-1}$ are uniquely defined as minimal nonnegative matrices which satisfy:

$$
\left\{\begin{array}{l}
\boldsymbol{R}_{N-1}=\mathbf{A}_{\mathbf{N}}+\boldsymbol{R}_{N-1} \mathbf{A}_{1}+\boldsymbol{R}_{N-1} \boldsymbol{R}_{1} \mathbf{A}_{0} \\
\boldsymbol{R}_{l}=\mathbf{A}_{1+1}+\boldsymbol{R}_{l} \mathbf{A}_{1}+\left[\boldsymbol{R}_{l} \boldsymbol{R}_{1}+\boldsymbol{R}_{l+1}\right] \mathbf{A}_{0}
\end{array} \quad \text { if } 1 \leq l \leq N-2\right.
$$

By collecting blocks of $(N-1) \times(N-1)$ matrices $\mathbf{A}_{\mathbf{i}}$ (starting at the top left corner of $\mathbf{P}$ ) and naming these blocks $\mathbf{B}^{\prime}, \mathbf{A}_{\mathbf{0}}^{\prime}, \mathbf{A}_{\mathbf{1}}^{\prime}$ and $\mathbf{A}_{\mathbf{2}}^{\prime}$, one can reduce the structure of $\mathbf{P}$ to a quasi-birth-and-death process as in (Neuts 1981). The equilibrium distribution $\underline{\pi}=\left[\begin{array}{llll}\underline{\pi}_{0}^{\prime} & \underline{\pi}_{1}^{\prime} & \underline{\pi}_{2}^{\prime} & \ldots\end{array}\right]$ where the $(N-1) M^{N}$-dimensional row vector $\underline{\pi}_{i}^{\prime}=\left[\underline{\pi}_{i(N-1)} \underline{\pi}_{i(N-1)+1} \cdots \underline{\pi}_{i(N-1)+N-2}\right]$ can be written in matrix geometric form (Neuts 1981):

$\underline{\pi}_{n}^{\prime}=\underline{\pi}_{0}^{\prime} \boldsymbol{R}^{n} \quad n=0,1, \ldots$

where $\boldsymbol{R}$ is the $(N-1) M^{N} \times(N-1) M^{N}$-dimensional rate matrix which is the minimum nonnegative solution of the following set of nonlinear equations:

$\boldsymbol{R}=\mathbf{A}_{\mathbf{2}}^{\prime}+\boldsymbol{R} \mathbf{A}_{1}^{\prime}+\boldsymbol{R}^{2} \mathbf{A}_{0}^{\prime}$

In both cases $\underline{\pi}_{0}^{\prime}=\left[\begin{array}{llll}\underline{\pi}_{0} & \underline{\pi}_{1} & \ldots & \underline{\pi}_{N-2}\end{array}\right]$ follows from the boundary equations $\underline{\pi}_{0}^{\prime} \mathbf{B}^{\prime}+\underline{\pi}_{1}^{\prime} \mathbf{A}_{0}^{\prime}=\underline{\pi}_{0}^{\prime}$ and the normalization equation $\sum_{i=0}^{\infty} \underline{\pi}_{i} \cdot \underline{e}=1$.

The main limitation of the previous methods is that for values of $N$ and $M$ which would model a realistic ATM multiplexer the number of equations in (5) or (7) becomes prohibitive, even though (5) and (7) can be solved iteratively. $N=20$ video sources each with a 10 -dimensional modulating state is a realistic example, but gives $10^{20}$ nonlinear equations to be solved! The main contribution of this paper consists in showing that by using the internal Kronecker product structure (2) of the A-matrices, a relation between the $\boldsymbol{R}_{\boldsymbol{i}}$ in (5) and the $\boldsymbol{R}$-matrix in (7) and by using the eigenvalue decomposition for $\boldsymbol{R}$, it is possible to reduce (5) or (7) to $N+1$ nonlinear equations in an eigenvalue of $\boldsymbol{R}$ and $N$ dummy variables. These equations are the same for all eigenvalues.

The relationship between the 2 methods above is shown by the following equality:

$$
\boldsymbol{R}=\left(\boldsymbol{R}^{\prime}\right)^{N-1}
$$


where $\boldsymbol{R}^{\prime}$ is a matrix with the same dimensions as $\boldsymbol{R}$, defined as

$$
\boldsymbol{R}^{\prime}=\left(\begin{array}{cccccc}
\mathbf{0} & \mathbf{0} & \mathbf{0} & \ldots & \mathbf{0} & \boldsymbol{R}_{N-1} \\
\mathbf{I} & \mathbf{0} & \mathbf{0} & \ldots & \mathbf{0} & \boldsymbol{R}_{N-2} \\
\mathbf{0} & \mathbf{I} & \mathbf{0} & \ldots & \mathbf{0} & \boldsymbol{R}_{N-3} \\
\mathbf{0} & \mathbf{0} & \mathbf{I} & \ldots & \mathbf{0} & \boldsymbol{R}_{N-4} \\
\vdots & \vdots & \vdots & & \vdots & \vdots \\
\mathbf{0} & \mathbf{0} & \mathbf{0} & \ldots & \mathbf{I} & \boldsymbol{R}_{1}
\end{array}\right)
$$

From (8) it follows that $\boldsymbol{R}$ and $\boldsymbol{R}^{\prime}$ have the same eigenvectors and that the eigenvalues of $\boldsymbol{R}$ are the $(N-1)$-th power of the eigenvalues of $\boldsymbol{R}^{\prime}$. To keep the notation simple, we assume that $\boldsymbol{R}^{\prime}$ is diagonalisable. In the general case Jordan forms have to be considered leading to very tedious calculations.

Substituting (9) in the eigenvalue equation $\underline{v} \boldsymbol{R}^{\prime}=s \underline{v}$ shows that each left eigenvector $\underline{v}$ (corresponding to eigenvalues $s_{i}, i=1, \ldots,(N-1) M^{N}$ ) has the following structure:

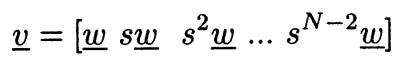

where the $M^{N}$-dimensional vectors $\underline{w}$ satisfies

$\underline{w}\left[\boldsymbol{R}_{N-1}+s \boldsymbol{R}_{N-2}+\ldots+s^{N-2} \boldsymbol{R}_{1}\right]=s^{N-1} \underline{w}$

Moreover the eigenvalues $s_{i}$ are the $(N-1) M^{N}$ roots of the polynomial equation

$\operatorname{det}\left[\boldsymbol{R}_{N-1}+s \boldsymbol{R}_{N-2}+\ldots+s^{N-2} \boldsymbol{R}_{1}-s^{N-1} \mathbf{I}\right]=0$

Unfortunately we cannot use (11) and (12) to solve the eigenvalue problem, since the rate matrices $\boldsymbol{R}_{\boldsymbol{i}}$ are unknown. Left multiplying (7), with the left eigenvector $\underline{v}$ expressed via (10), we find that $\underline{w}$ must satisfy

$\underline{w} \mathbf{X}(s)=\underline{w}\left[\mathbf{A}_{\mathbf{N}}+s \mathbf{A}_{\mathbf{N}-\mathbf{1}}+s^{2} \mathbf{A}_{\mathbf{N}-2}+\ldots+s^{N-1} \mathbf{A}_{\mathbf{1}}+s^{N} \mathbf{A}_{\mathbf{0}}\right]=s^{N-1} \underline{w}$

A necessary condition for $s$ to be an eigenvalue is that $\operatorname{det}\left(\mathbf{X}(s)-s^{N-1} \mathbf{I}\right)=0$. This characteristic equation is a polynomial of degree between $(N-1) M^{N}$ and $N M^{N}$, depending on the rank of $\mathbf{A}_{\mathbf{0}}$. This means that the characteristic equation can have more than $(N-1) M^{N}$ roots, but we can show that there are exactly $(N-1) M^{N}$ solutions of $\operatorname{det}\left(\mathbf{X}(s)-s^{N-1} \mathbf{I}\right)=0$ which satisfy $0 \leq|s|<1$; these are the $(N-1) M^{N}$ eigenvalues of $\boldsymbol{R}$. This can be proven as follows: in (Neuts 1981) it is shown that all $(N-1) M^{N}$ eigenvalues of $\boldsymbol{R}$ lie inside the unit circle, as well as all $M^{N}$ eigenvalues of $\boldsymbol{R}_{\beta}$, as defined in section 6 of this paper and in (Wuyts and Boel 1996). If the average load of 
the buffer is less than 1, exactly one eigenvalue of $\boldsymbol{R}_{\beta}$ will be 1 , the others lie strictly inside the unit circle. In section 6 it is also shown that the finite inverses of the eigenvalues of $\boldsymbol{R}_{\beta}$ satisfy the same characteristic equation as the eigenvalues of $\boldsymbol{R}$. We say only the finite inverses, because some of the eigenvalues of $\boldsymbol{R}_{\boldsymbol{\beta}}$ (let us say $k$ in total) might be 0 (because the rank of $\mathbf{A}_{\mathbf{0}}$ equals $\left.M^{N}-k\right)$. This means that $\operatorname{det}\left(\mathbf{X}(s)-s^{N-1} \mathbf{I}\right)=0$ has $(N-1) M^{N}$ roots inside the unit circle (i.e. the eigenvalues of $\boldsymbol{R}$ ) and $M^{N}-k$ finite roots outside the unit circle (i.e. the inverse of the eigenvalues of $\boldsymbol{R}_{\beta}$ which are different from 0 ), including the root at 1.

Because of the Kronecker product structure of $\mathbf{A}_{\mathbf{i}}$ in (2), $\mathbf{X}(s)$ can be decomposed as $\mathbf{X}(s)=\mathbf{Q}_{\mathbf{1}}(s) \otimes \mathbf{Q}_{\mathbf{2}}(s) \otimes \ldots \otimes \mathbf{Q}_{\mathbf{N}}(s)$ where $\mathbf{Q}_{\mathbf{i}}(s)=\left(\mathbf{P}_{\mathbf{i}}+s\left(\mathbf{I}-\mathbf{P}_{\mathbf{i}}\right)\right) \mathbf{Q}_{\mathbf{i}}$. Let us assume that $\underline{w}$ also has a Kronecker product structure, then a sufficient condition for $\underline{w}$ and $s$ to satisfy (13) is

$$
\begin{aligned}
& \underline{x}_{i} \mathbf{Q}_{\mathbf{i}}(s)=\mu_{i} \underline{x}_{i} \quad i=1, \ldots, N \\
& \prod_{i=1}^{N} \mu_{i}=s^{N-1} \\
& \underline{w}=\underline{x}_{1} \otimes \underline{x}_{2} \otimes \ldots \otimes \underline{x}_{N}
\end{aligned}
$$

Notice that $\underline{x}_{i}$ is an M-dimensional vector and every $\mu_{i}$ is a dummy variable, used only in order to decompose one large set (13) of $M^{N}$ linear equations in $\underline{w}$ into $N$ smaller sets (14) of $M$ linear equations in $\underline{x}_{i}$. The solution for $s$ and $\mu_{i}$ is given by the condition that the solution for each $\underline{x}_{i}$ must differ from 0 . This yields the following set of $N+1$ nonlinear equations:

$\operatorname{det}\left(\mathbf{Q}_{\mathbf{i}}(s)-\mu_{i} \mathbf{I}\right)=0 \quad i=1, \ldots, N$

$\prod_{i=1}^{N} \mu_{i}=s^{N-1}$

The first $N$ equations (17) are decoupled per source, since each equation only contains the variable $s$ and 1 variable $\mu_{i}$. So the set is only coupled through the last equation (18).

\section{THE EQUILIBRIUM DISTRIBUTION}

If $\boldsymbol{R}^{\prime}$ is diagonalisable, its set of left eigenvalues $\underline{v}_{i}$ (as given by (10)) is a base for the $(N-1) M^{N}$-dimensional space. So also $\underline{\pi}_{0}^{\prime}$ can be written as a linear combination of these eigenvectors $\underline{v}_{i}$ and this yields a general expression for 
the equilibrium distribution $\underline{\pi}$ :

$\underline{\pi}_{n}=\sum_{i=1}^{(N-1) M^{N}} b_{i} s_{i}^{n} \underline{w}_{i} \quad n=0,1,2, \ldots$

The unknown scalars $b_{i}$ are uniquely determined by the boundary equations $\underline{\pi}_{0}^{\prime} \mathbf{B}^{\prime}+\underline{\pi}_{1}^{\prime} \mathbf{A}_{0}^{\prime}=\underline{\pi}_{0}^{\prime}$ and the normalization equation $\sum_{i=0}^{\infty} \underline{\pi}_{i} \cdot \underline{e}=1$. Substitution of (19) in both equations yields

$\underline{b} \mathbf{W} \mathbf{U}=0$

$\sum_{i=1}^{(N-1) M^{N}} \frac{b_{i}}{1-s_{i}} \underline{w}_{i} \underline{e}=1$

and $\mathbf{W}, \mathbf{U}$ and $\underline{b}$ are given by

$\underline{b}=\left[b_{1} b_{2} \ldots b_{(N-1) M^{N}}\right]$

$\mathbf{W}=\left(\begin{array}{cccc}\underline{w}_{1} & s_{1} \underline{w}_{1} & \ldots & \underline{s}_{2}^{N-1} \underline{w}_{1} \\ \underline{w}_{2} & s_{2} \underline{w}_{2} & \cdots & s_{2}^{N-1} \underline{w}_{2} \\ \vdots & \vdots & & \vdots \\ \underline{w}_{(N-1) M^{N}} & s_{(N-1) M^{N} \underline{w}_{(N-1) M^{N}}} & \ldots & s_{(N-1) M^{N}}^{N-1} \underline{w}_{(N-1) M^{N}}\end{array}\right)$

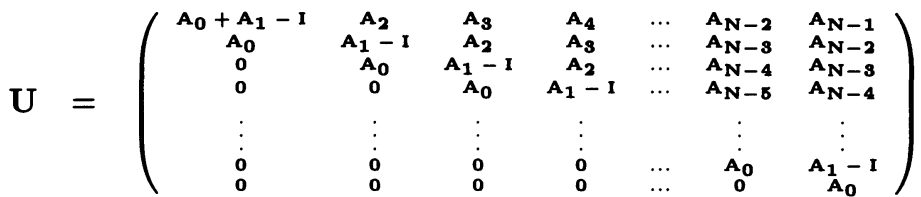

Notice that the computation of $\underline{b}$ still involves solving a set of $(N-1) M^{N}$ linear equations in the same amount of variables. For now we suggest to solve this set using the classical methods, such as LU-decomposition, but the structure of $\mathbf{W}$ and $\mathbf{U}$ suggests that there exist more efficient methods, allowing us to compute equilibrium distributions for larger $N$ and $M$. Research on this subject is in progress.

\section{A B-ISDN EXAMPLE : COMPUTATION OF THE EIGENVALUES}

If the eigenvalues $s_{i}$ and the corresponding dummy variables are known, the left eigenvectors $\underline{w}_{i}$ can be easily computed in (14) and (16), and also all $b_{i}$ follow from a set of linear equations (20)-(21). Only the numerical computation 
of the eigenvalues needs further explanation, since the equations (17)-(18) are not linear.

Let us first assume that the transition matrix $\mathbf{Q}_{\mathbf{i}}$ of the modulating Markov process for source $i$ has the following structure:

$\mathbf{Q}_{\mathbf{i}}=\left(\begin{array}{ccccccc}1-\sum_{j=2}^{M} \alpha_{j}(i) & \alpha_{2}(i) & \alpha_{3}(i) & \alpha_{4}(i) & \ldots & \alpha_{M-1}(i) & \alpha_{M^{(i)}} \\ \beta_{2}(i) & 1-\beta_{2}(i) & 0 & 0 & \ldots & 0 & 0 \\ \beta_{3}(i) & 0 & 1-\beta_{3}(i) & 0 & \ldots & 0 & 0 \\ \vdots & \vdots & \vdots & \vdots & & \vdots & \vdots \\ \beta_{M}(i) & 0 & 0 & 0 & \ldots & 0 & 1-\beta_{M^{(i)}}\end{array}\right)$

It is shown in (Robert and Le Boudec "Can..." 1995) and (Robert and Le Boudec "Stoch..." 1995) that this assumption provides a good model for VBR data sources on ATM networks such that long range dependence of these sources is approximated very well over several orders of magnitude of time. The time interval over which the long range dependent behaviour of this model is valid can be increased by increasing the number $M$ of states per source. Also in the EXPERT-project experiments have shown that the assumed structure for $\mathbf{Q}_{\mathbf{i}}$ gives a good model for VBR video over ATM (ACTS 1997). It has been observed that cell losses depend strongly on correlations between cell arrivals during bursts of high rates. These bursts are generated by states $j$ such that $\alpha_{j}(i)$ and $\beta_{j}(i)$ are small and the diagonal elements of $\mathbf{Q}_{\mathbf{i}}$ close to 1 .

Another advantage of (25) is that the determinant of (17) is easy to compute:

$$
\begin{aligned}
& \operatorname{det}\left(\mathbf{Q}_{\mathbf{i}}(s)-\mu_{i} \mathbf{I}\right)=a_{1}(i) \prod_{k=2}^{M} c_{k}(i)-\left[\sum_{j=2}^{M} a_{j}(i) b_{j}(i) \prod_{k=2, k \neq j}^{M} c_{k}(i)\right] \\
& c_{k}(i)=\left(p_{k}(i)+s\left(1-p_{k}(i)\right)\right)\left(1-\beta_{k}(i)\right)-\mu_{i} \quad k=2, \ldots, M \\
& b_{j}(i)=\left(p_{j}(i)+s\left(1-p_{j}(i)\right)\right) \beta_{j}(i) \quad j=2, \ldots, M \\
& a_{j}(i)=\left(p_{1}(i)+s\left(1-p_{1}(i)\right)\right) \alpha_{j}(i) \quad j=2, \ldots, M \\
& a_{1}(i)=\left(p_{1}(i)+s\left(1-p_{1}(i)\right)\right)\left(1-\sum_{j=2}^{M} \alpha_{j}(i)\right)-\mu_{i}
\end{aligned}
$$

where $p_{k}(i)$ is the $\mathrm{k}$-th diagonal element of $\mathbf{P}_{\mathbf{i}}$. It is obvious that the determinant in (17) is a polynomial of degree $M$ in $\mu_{i}$ as well as in $s$. We want to emphasize here that the structure in (25) is not a necessary condition for the validity of the method described in this paper. The method is still valid for a general transition matrix $\mathbf{Q}_{\mathbf{i}}$. The only necessary condition for the validity 
of (31)-(32) is the Kronecker product structure in (2), in other words: the arrival stream must consist of independent Markovian processes. The structure in (25) is only chosen to simplify the computation of the determinant in (31) and because this structure is known to occur in practical situations.

The numerical method for the computation of $s$ is based upon repeated substitution. The initial step is $s^{(0)}=0$. The iterative algorithm is given by

$$
\begin{aligned}
& \operatorname{det}\left(\mathbf{Q}_{\mathbf{i}}\left(s^{(k)}\right)-\mu_{i}^{(k+1)} \mathbf{I}\right)=0 \quad i=1, \ldots, N \\
& \left(s^{(k+1)}\right)^{N-1}=\prod_{i=1}^{N} \mu_{i}^{(k+1)}
\end{aligned}
$$

Since (31) is a polynomial of degree $M$ in $\mu_{i}^{(k+1)}$, we see that $\mu_{i}^{(k+1)}$ is not a function of $s^{(k)}$, unless we order the $M$ roots of the polynomial (e.g. with increasing real part), give these roots indices from 1 to $M$ and during each iteration choose the root with the same index. We do this for all $N$ equations and substitute the solutions in the right member of (32). Again $s^{(k+1)}$ is not a function of the product in the right member of $(32)$, since the $(N-1)$ th root has $N-1$ (complex) solutions. Also here we can order the roots with increasing angles with respect to the real axis in the complex plane, give indices from 1 to $N-1$ and again choose the root with the same index during every iteration step. Notice that the $N$ polynomial equations in (31) all have $M$ roots such that the number of combinations in the product in (32) equals $M^{N}$. For each of these $M^{N}$ combinations there are $N-1$ roots $s$, so we find $(N-1) M^{N}$ solutions for $s$, which is exactly the number of solutions for $s$ within the unit circle.

Although the iterative algorithm converges to the correct solution in most cases, it can get caught in a periodic cycle in some cases. During tests we noticed that this periodic cycle in the case of nonconvergence always passed the neighbourhood of the correct solution. This suggests the following extension of the iterative method with a minimization algorithm. Let $\mu_{i, l}(s)$ denote the solution $\mu_{i}$ of the $i$ th equation in (17) with the $l$ th largest real part, such that $\mu_{i, l}(s)$ is a well defined function of $s$. In that case we can define the following squared error function:

$\epsilon^{2}(s)=\left|\prod_{i=1}^{N} \mu_{i, l(i)}(s)-s^{N-1}\right|^{2}$

It is obvious that the minima of $\epsilon^{2}(s)$, which equal 0 , are reached at the solutions $s$ of (17)-(18). If the iterative method (31)-(32) does not converge, it is possible to compute $\epsilon^{2}(s)$ during every iteration step and find the neighbourhood of the correct root by choosing the minimum in the periodic cycle. 
It has been found experimentally that Powell's Direction Set method (Press et al. 1989) always finds the correct minimum.

The following table shows the eigenvalues $s_{i}$ for $N=4$ identical sources with $M=2$ states per source (on/off-sources) with $\alpha_{2}=0.04, \beta_{2}=0.01, p_{1}=0.95$ and $p_{2}=0.01$ such that the average load $\rho=0.792$.

\begin{tabular}{ccc}
\hline eigenvalues & multiplicity & convergence \\
\hline 0.003007288 & 1 & $\mathrm{y}$ \\
0.2067359 & 4 & $\mathrm{y}$ \\
0.8951560 & 6 & $\mathrm{y}$ \\
0.9425859 & 4 & $\mathrm{y}$ \\
0.9842921 & 1 & $\mathrm{y}$ \\
$-0.001208725+\mathrm{j} 0.001361218$ & 1 & $\mathrm{y}$ \\
$-0.004877811+\mathrm{j} 0.002913184$ & 4 & $\mathrm{y}$ \\
$-0.009916731+\mathrm{j} 0.001100736$ & 6 & $\mathrm{n}$ \\
-0.8027469 & 4 & $\mathrm{y}$ \\
$-0.4659548+\mathrm{j} 0.7183651$ & 1 & $\mathrm{y}$ \\
$-0.001208725-\mathrm{j} 0.001361218$ & 1 & $\mathrm{y}$ \\
$-0.004877811-\mathrm{j} 0.002913184$ & 4 & $\mathrm{y}$ \\
$-0.009916731-\mathrm{j} 0.001100736$ & 6 & $\mathrm{n}$ \\
-0.01010240 & 4 & $\mathrm{n}$ \\
$-0.4659548-\mathrm{j} 0.7183651$ & 1 & $\mathrm{y}$ \\
\hline
\end{tabular}

Since we consider identical sources, there are only 15 different eigenvalues, instead of $(N-1) M^{N}=48$, but some of them have a multiplicity larger than 1 . The matrix $\boldsymbol{R}^{\prime}$ is still diagonalizable, because every eigenvalue has as many different eigenvectors as its multiplicity. The reason can be found in (14) and (16) : the $N=4$ equations in (14) are now identical but depending on which of the roots $\mu_{i}$ we choose to substitute in the product in (15), the eigenvectors $\underline{x}_{i}$ can still be different. Taking permutations of the Kronecker product in (16) gives different vectors $\underline{w}$ corresponding to the same eigenvalue $s$. The number of these permutations is the multiplicity of $s$ and equals $C_{N}^{l}$ for $M=2 ; l$ (or $N-l$ ) is the number of variables $\mu_{i}$ which have the same value in the product of (15).

In the table we also pointed out for which eigenvalues the repeated substitution does not converge. Notice that nonconvergence only occurs for 3 of the 15 eigenvalues which are very close to each other, such that the squared error function has very steep slopes in the neighbourhood of these values. Nevertheless Powell's Direction Set method has no problem converging to the correct solutions after using repeated substitution to come in their neighbourhood.

Figures 1 and 2 show the stationary distribution for the buffer occupancy (as a measure for the delay characteristics) and the CLR for the same example as discussed above. The figures compare the results for the finite and infinite 
buffer case. In the infinite buffer case the figures show several curves, taking into account different numbers of eigenvalues. It can be seen that reliable estimates of the CLR can be obtained using only a fraction of the eigenvalues for the computation of the results, neglecting the other eigenvalues as if they were 0 . The curves taking into account all eigenvalues show the exact results. The other curves take into account only the positive real eigenvalues (the computation of these is always stable) or only 1 eigenvalue (the maximum positive real eigenvalue). Particularly the curves for the CLR are close to each other, which implies that not all eigenvalues should be computed.

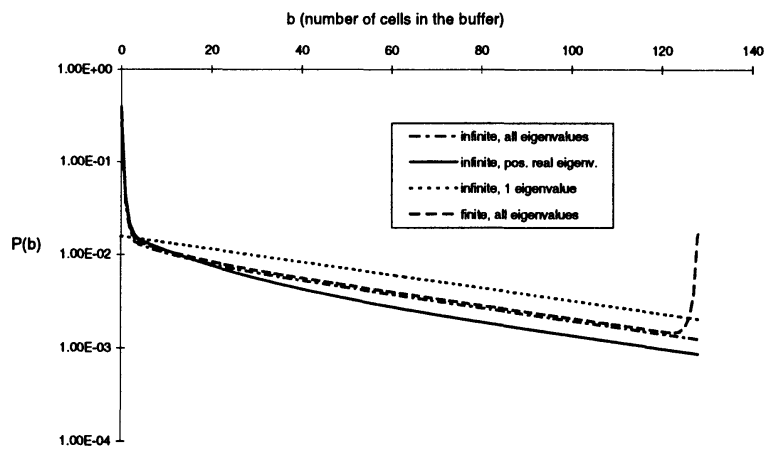

Figure 1 The probability $\mathrm{P}(\mathrm{b})$ that there are $\mathrm{b}$ cells in the buffer as a function of $\mathrm{b}$ for $\mathrm{N}=4$ identical sources with $\mathrm{M}=2$ states per source and $\mathrm{B}=128$; $\alpha_{2}=0.04, \beta_{2}=0.01, p_{\text {on }}=0.95$ and $p_{\text {off }}=0.01, \rho=0.792$.

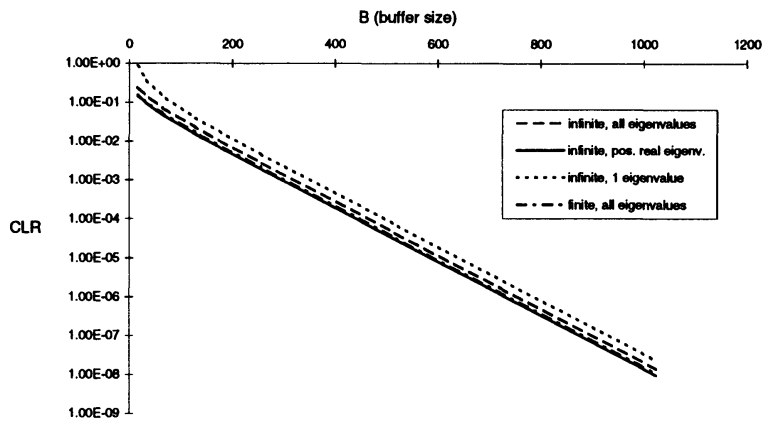

Figure $2 \mathrm{CLR}$ as a function of buffer size $\mathrm{B}$ for $\mathrm{N}=4$ identical sources with $\mathrm{M}=2$ states per source; $\alpha_{2}=0.04, \beta_{2}=0.01, p_{\text {on }}=0.95$ and $p_{\text {of } f}=0.01, \rho=0.792$.

Current research involves finding heuristic rules for deciding which eigenvalues to compute. In any case it is obvious that good approximates can be obtained using a small number of eigenvalues, but that moreover a significant improve- 
ment (figures 1 and 2 have a logarithmic scale) can be achieved by using more than one eigenvalue.

\section{EXTENSION OF THE ALGORITHM TO THE FINITE BUFFER CASE}

In (Wuyts and Boel 1996) it is shown that the stationary probability distribution for a finite buffer is the superposition of 2 waves: $\underline{\pi}_{n}=\underline{\alpha}_{n}+\underline{\beta}_{n}$. Indeed in the finite buffer case (3) can be interpreted as a linear difference equation with boundary conditions at the left and the right boundary. The general solution can be written as a superposition of the responses to respectively the left and the right boundary conditions. A physical analogon is as follows. The first wave $\underline{\alpha}_{n}$ propagates from left to right and carries the effects of an empty buffer, while $\beta_{n}$ propagates from right to left and carries the effects of a full buffer. The first term $\underline{\alpha}_{n}$ is created by the same equations (4) and (5) as for $\underline{\pi}_{n}$ in the infinite buffer case. This means that the spectral analysis for $\underline{\alpha}_{n}$ is the same as described above. The second term is given by $\underline{\beta}_{B-n}=\underline{\beta}_{B} \boldsymbol{R}_{\beta}{ }^{n}$ where $B$ is the length of the finite buffer. The rate matrix $\boldsymbol{R}_{\beta}$ fulfills the following equation:

$\boldsymbol{R}_{\beta}=\mathbf{A}_{\mathbf{0}}+\boldsymbol{R}_{\beta} \mathbf{A}_{1}+\boldsymbol{R}_{\beta}{ }^{2} \mathbf{A}_{2}+\ldots+\boldsymbol{R}_{\beta}{ }^{N} \mathbf{A}_{\mathbf{N}}$

This means that the eigenvectors $\underline{w}_{\beta}$ of the rate matrix $\boldsymbol{R}_{\beta}$ must satisfy $\underline{w}_{\beta} s_{\beta}^{N} \mathbf{X}\left(1 / s_{\beta}\right)=s_{\beta} \underline{w}_{\beta}$ such that the corresponding eigenvalues $s_{\beta}$ can be found as in (17)-(18), with substitution of $\mathbf{Q}_{\mathbf{i}}(s)$ in (17) by $\left(s_{\beta} \mathbf{P}_{\mathbf{i}}+\mathbf{I}-\mathbf{P}_{\mathbf{i}}\right) \mathbf{Q}_{\mathbf{i}}$ and with substitution of $s^{N-1}$ in (18) by $s_{\beta}$.

If the average load $\rho$ is less than 1 , one of the solutions $s_{\beta}$ equals 1 . The other eigenvalues lie inside the unit circle, and when constructed correctly they must equal the inverse $1 / s$ of the solutions of $\operatorname{det}\left(\mathbf{X}(s)-s^{N-1} \mathbf{I}\right)=0$ which lie on or outside the unit circle. Nevertheless when using repeated substitution the convergence will be better when looking for solutions within the unit circle, and therefore the equations within section 6 should be used instead of the equations in the previous sections.

\section{CONCLUSION}

The main contribution of this paper is to reduce further the computational burden for a technique, introduced in (Wuyts and Boel 1996), to compute the CLR and delay characteristics for realistic VBR arrival traffic in an ATM buffer. In (Wuyts and Boel 1996) the algorithm involves the iterative computation of $N-1$ rate matrices of dimensions $M^{N} \times M^{N}$ (for an infinite buffer). The algorithm introduced in this paper computes the $(N-1) M^{N}$ 
eigenvalues and the corresponding left eigenvectors of 1 larger matrix, containing all these rate matrices, without having to compute this matrix itself. All the eigenvalues can be obtained by finding all solutions of a set of $N+1$ nonlinear equations in $s$ and in $N$ dummy variables. The first $N$ equations are decoupled per source in the sense that they contain only $s$ and 1 dummy variable. The last equation contains all $N+1$ variables.

A further reduction of the dimensions might be obtained by computing only a fraction of the $(N-1) M^{N}$ terms in (19). When computing e.g. the CLR we are only interested in the values of the equilibrium distribution close to a full buffer. Many of the terms in (19) are usually so small in the full buffer region that they can be neglected. This happens e.g. for the terms corresponding to eigenvalues $s$ with a small amplitude. During the iteration step (31) we have to choose which of the solutions of $\mu_{i}$ we have to substitute in (32). By choosing the solutions with the largest amplitudes we find the eigenvalue with the largest amplitude, etc. This means that we only have to compute a fraction of the eigenvalues. Unfortunately, depending on the length of the buffer some of the small eigenvalues might not be negligible because the corresponding values of $b_{i}$ in (19) might be large such that the corresponding terms are still substantial in the full buffer region. The selection of which eigenvalues to compute is still a topic of current research.

\section{REFERENCES}

ACTS 094 EXPERT (1997) First results for trials of optimised traffic control features. ACTS 094 EXPERT, DELIVERABLE 10, WPG4, 44-63.

Blondia, C. and Casals, O. (1992) Statistical multiplexing of VBR sources : a matrix-analytic approach. Performance Evaluation, 16, 5-20.

Hajek, B. (1982) Birth-and-Death processes on the integers with phases and general boundaries. J. Appl. Prob., 19, 488-499.

Latouche, G. and Ramaswami, V. (1993) A logarithmic reduction algorithm for Quasi Birth and Death processes. J. Appl. Prob., 30, 650-674.

Mitrani, I. and Chakka, R. (1995) Spectral expansion solution for a class of Markov models: application and comparison with the matrix-geometric method. Performance Evaluation, 23, 241-260.

Naoumov, V., Krieger, U. and Wagner, D. (1996) Analysis of a multi-server delay-loss system with a general Markovian arrival process. Matrix analytic methods in stochastic models, Marcel Dekker, New York, 4366.

Neuts, M.F. (1981) Matrix-geometric solutions in stochastic models. The Johns Hopkins University Press, Baltimore.

Press, W.H., Flannery B.P., Teukolsky, S.A. and Vetterling W.T. (1989) Numerical recipes. Cambridge University Press, Cambridge.

Robert, S. and Le Boudec, J. (1995) Can self-similar traffic be modeled by Markovian processes? COST-242 report. 
Robert, S. and Le Boudec, J. (1995) Stochastic processes for self-similar traffic. COST-242 report.

Wuyts K. and Boel R.K. (1996) A matrix geometric algorithm for finite buffer systems with B-ISDN applications. Proceedings of the ITC Specialists Seminar on Control in Communications, September 17-19, Lund, Sweden, 265-276.

Xiong, Y. and Bruneel, H. (1996) A unifying queueing model for ATM and its analysis. International Journal of Communication Systems, 9, 253267.

Ye, J. and Li, S. (1994) Folding algorithm : a computational method for finite QBD processes with level-dependent transitions. IEEE Transactions on Communications, 42, 625-639.

\section{BIOGRAPHY}

Kristiaan Wuyts graduated as Electrotechnical Engineer at the University of Ghent (Belgium) in 1991. In the same year he started as a research assistant at the Department of Telecommunication and Information Processing of the same university, where he worked on modulation and coding theory. In 1995 he changed to the SYSTeMS Department where he is currently working as research assistant and $\mathrm{PhD}$ student. His main interests are queueing theory, performance analysis, ATM networks and computer systems.

René K. Boel was born in Aalst, Belgium, on June 15, 1946. He received the electro-mechanical engineering degree and the nuclear engineering degree from the State University at Ghent, Belgium, in 1969 and 1970, and the M.Sc. and $\mathrm{Ph}$. D. degrees from the University of California, Berkeley, in 1972 and 1974 resp. He is a Research Fellow of the F.W.O.-Vlaanderen (Flemish Foundation for Scientific Research) at the Department of Electrical Engineering of the University at Ghent, and an Associate Professor (part-time) at the Department of Mathematics of the Katholieke Universiteit Leuven. He has held temporary appointments at various universities abroad. His current research interests are in stochastic control and its applications to communications networks, and in control of discrete event and hybrid systems. 